\title{
1 Blutgerinnung und Antikoagulation
}

\author{
Claus Steuernagel
}

Adipositas gilt als relevanter Risikofaktor für das Auftreten kardiovaskulärer Erkrankungen. Mediatoren und Hormone, freigesetzt aus Adipozyten, nehmen einen starken Einfluss auf verschiedene Stoffwechselvorgänge, so auch auf die Hämostase. Bei Adipositas besteht ein 2- bis 3-fach erhöhtes Risiko, eine thromboembolische Komplikation zu erleiden. Physiologischerweise sorgt das ausgewogene Zusammenspiel prokoagulatorischer und antikoagulatorischer Systeme bei einer Verletzung für eine lokale Blutstillung, ohne dass Gerinnungsthromben außerhalb der Verletzungsstelle entstehen. Bei Adipositas kann diese Balance gestört sein. Die Kombination von gesteigerter Thrombozytenaktivität und verminderter Fibrinolyse kann zu einem lokal unkontrollierten Thrombuswachstum führen und das Auftreten von tiefer Venenthrombose und Lungenembolie begünstigen.

Bei Adipositas besteht kein relevant erhöhtes hämostaseologisch bedingtes Blutungsrisiko. Dies spiegelt sich beispielsweise darin wider, dass bei Übergewichtigen, Fettleibigen und schwerst Fettleibigen signifikant seltener transfundiert werden muss (Nafiu et al. 2011).

\subsection{Physiologie der Hämostase in vivo}

Seit Ende der 1990er-Jahre ist allgemein anerkannt, dass das bis dato angewand te Gerinnungsmodell mit intrinsischem und extrinsischem Weg heutzutage nur für die Interpretation von Laborwerten (z.B. Quick-Wert und aPTT) herangezogen werden sollte, nicht jedoch die in vivo-Situation der Hämostase wiedergeben kann. Es wird hierfür das sogenannte zellbasierte Modell verwendet, das nur einen einzigen Aktivierungsweg kennt, der dem extrinsischen Weg aus dem früheren Modell ähnelt (Hofmann u. Monroe 2001).

Die in vivo parallel ablaufenden Prozesse der Thrombozytenaggregation (primäre Hämostase) und der Fibrinbildung werden hier aus didaktischen Gründen teilweise nacheinander dargestellt.

\section{Primäre Hämostase}

Bei einer Gewebs- und Gefäßverletzung nähern sich Thrombozyten dem freiliegenden Kollagen des Blutgefässes. Vermittelt über den von-Willebrand-Faktor kommt es hier zur Adhäsion der Thrombozyten an das subendotheliale Kollagen. Hierdurch werden die Thrombozyten aktiviert und setzen prokoagulatorische Substanzen aus ihren Granula frei (u.a. Thromboxan $A_{2}$, von-Willebrand-Faktor, Fibrinogen etc.). Dies führt zu einer verstärkten Aktivierung der adhädierenden Thrombozyten, wodurch nun auf deren Oberfläche Rezeptoren für die Bindung von Fibrinogen exprimiert werden (GP IIb-IIIa-Rezeptoren). Fibrinogen bindet an benachbarte Thrombozyten und vermittelt so deren Aggregation. Die Aktivierung der Thrombozyten führt darüber hinaus zur Expression von Phospholipiden auf ihrer Oberfläche, wo Gerinnungsfaktoren binden können. 


\section{Plasmatische Gerinnung}

Die sogenannte plasmatische Gerinnung wird in vivo am subendothelialen Kollagen initialisiert und läuft anschließend auf der Thrombozytenoberfläche ab. Im Bereich einer vaskulären Verletzungsstelle wird tissue factor (syn. Gewebethromboplastin) exprimiert. Gebunden an tissue factor katalysiert Faktor VIIa die Aktivierung der Faktoren X und IX. Diese Initialisierung wird in vivo meist nach etwa einer Minute durch die Aktivität von tissue factor pathway inhibitor (tfpi) beendet, wodurch der Gerinnungsprozess nun auf die Oberfläche der Thrombozyten verlagert wird. Die zu diesem Zeitpunkt gebildete Menge an Thrombin reicht gerade aus, um den Quick-Wert normal ausfallen zu lassen. Für eine ausreichende Fibrin-Bildung und Aktivierung der Thrombozyten müssen weitere Prozesse (Amplifikation und Propagation) ablaufen, die zur Bildung einer größeren Menge an Thrombin führen.

Bei der Amplifikation führt die Aktivität der Faktoren XIa, VIIIa und Faktor IXa über die Aktivierung des Faktor X zur Bildung einer größeren Menge Thrombin. Während der Propagation (engl. Vermehrung, Ausbreitung) spielt Thrombin die zentrale Rolle. Die große Menge an Thrombin bzw. die auf den Thrombozyten entstandene Aktivität dieses Enzyms reicht nun aus, um relevante Mengen an Fibrinogen in Fibrin umzuwandeln. Darüber hinaus bewirkt Thrombin eine starke Aktivierung von Thrombozyten, was zu deren Aggregation führt (siehe auch: Primäre Hämostase). Nach Bildung des Fibringerinnsels stabilisiert der Faktor XIIIa den Thrombus durch Vernetzung der Fibrinmoleküle.

\section{TAFI (thrombin activatable fibrinolysis inhibitor)}

Aktiviert durch Thrombin verhindert TAFI die Bindung von Plasminogen an das Fibrin-Molekül und verhindert dadurch die Entstehung des fibrinolytischen Enzyms Plasmin.

\section{PAl-1 (Plasminogen-Aktivator-Inhibitor 1)}

PAI-1, syn. Serpin E1, gilt als wichtigster Inhibitor der physiologisch initiierten Fibrinolyse durch tissue Plasminogen-Aktivator (t-PA). PAI-1 wird in Endothelzellen gebildet, zirkuliert im Blut und wird in Thrombozyten gespeichert. PAI-1 inhibiert t-PA durch Bildung eines stabilen Komplexes (Loskutoff u. Samad 1998), der anschließend durch Endozytose eliminiert wird (Binder et al. 2002). Durch die PAI-1vermittelte Inaktivierung von t-PA wird die Aktivierung von Plasminogen zu Plasmin blockiert und so die Fibrinolyse eines gebildeten Thrombus verhindert (s. Abb. 1). Thrombozyten können gespeicherten PAI1 bei ihrer Aktivierung im Rahmen der primären Hämostase freisetzen und so einen gerade gebildeten Thrombus vor dessen Auflösung schützen.

\subsection{Physiologie der Antikoagulation in vivo}

Die Blutgerinnung läuft dann optimal ab, wenn prokoagulatorische Prozesse auf den Ort einer Blutung bzw. Verletzung begrenzt bleiben und die Entstehung von Thromboembolien vermieden wird. Dies wird durch das Zusammenspiel mehrerer antikoagulatorisch wirksamer Prozesse bewirkt.

\section{Protein C-System (Hemmung prokoagulatorischer Prozesse)}

In der Nähe einer Verletzung exprimiert unverletztes Gefäßendothel, stimuliert durch Thrombin, den Endothelrezeptor Thrombomodulin, an dem nun Protein C aktiviert wird. Aktiviertes Protein C (APC) hemmt im Bereich des gesunden Endothels die Blutgerinnung, indem es die Gerinnungsfaktoren Va und VIIIa durch Proteolyse inaktiviert.

\section{Antithrombin (Inaktivierung von Gerinnungsfaktoren)}

Im Bereich einer vaskulären Verletzungsstelle reicht die Aktivität von Antithrombin kaum aus, um etwas gegen die hohe Konzentration aktivierter Gerinnungsfaktoren auszurichten. Im Abstromgebiet der Verletzungsstelle allerdings können abtransportierte Gerinnungsfaktoren durch Antithrombin inaktiviert werden, wodurch unphysiologisch ablaufende prokoagulatorische Prozesse außerhalb der Verletzungsstelle unterbunden werden.

\section{Fibrinolyse (Auflösung eines bereits entstandenen Blutgerinnsels)}

Parallel zur Initialisierung der Hämostase an einer vaskulären Verletzungsstelle wird auch das Fibrinolyse-System aktiviert. Dies dient dazu, überschießend abgelaufene prokoagulatorische Prozesse zu limitieren. Dies kommt durch die Expression von t-PA 


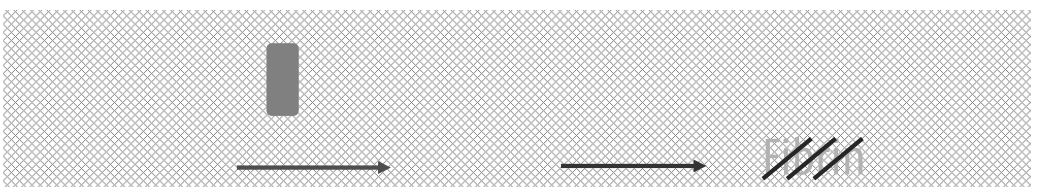

Physiologisch: Plasmin lysiert überschüssiges Fibrin

\section{Adipositas: PAl-1 blockiert t-PA $\rightarrow$ Thrombuswachstum}

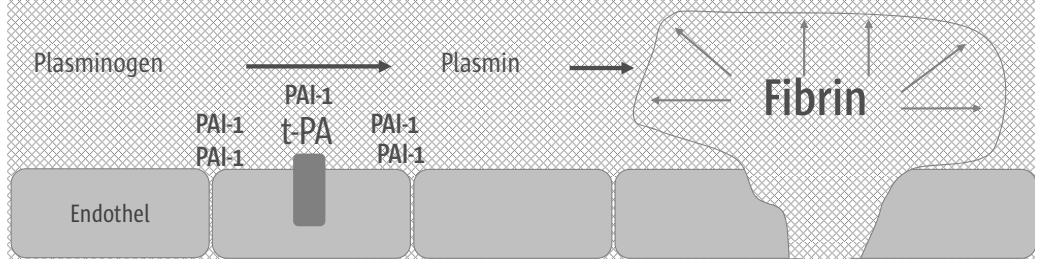

Abb. 1 Physiologischerweise lysiert Plasmin überschüssiges Fibrin und verhindert dadurch Thrombosen. Bei Adipositas droht ein gesteigertes Thrombuswachstum durch erhöhte Konzentrationen von PAl-1 (Plasminogen-Aktivator-Inhibitor 1). PAl-1 blockiert t-PA (tissue Plasminogen-Aktivator), woraus eine abgeschwächte Lyse von überschüssigem Fibrin resultiert.

(tissue plasminogen activator) auf dem Endothel in der Nähe der vaskulären Verletzungsstelle zustande. t-PA aktiviert Plasminogen zu Plasmin, welches dann das im Thrombus entstandene Fibrin in D-Dimere spaltet und so den Thrombus auflöst.

Würden die fibrinolytischen Prozesse auch direkt im Bereich der Verletzungsstelle ablaufen, dann bestünde die Gefahr, dass sich ein gerade gebildeter Thrombus wieder auflöst und so eine Blutung entsteht. Um genau dies zu verhindern, wird der Thrombus im Verletzungsbereich durch verschiedene Faktoren vor Lyse geschützt.

\subsection{Thromboseneigung und Adipositas}

Die pathologisch erhöhte Wirkung von PAI-1 gilt bei Adipositas als eine der Hauptursachen für das erhöhte thromboembolische Risiko (Loskutoff 1998). Adipozyten produzieren gesteigerte Mengen an PAI-1 (Loskutoff u. Samad 1998). Durch PAI-1 wird die Wirkung des Fibrinolyse-Aktivators t-PA herabgesetzt. Hierdurch werden gebildete Thromben nicht ausreichend in ihrem Wachstum begrenzt(s. Abb. 1), sodass die Entstehung einer tiefen Venenthrombose (TVT) gefolgt von einer Lungenarterienembolie (LE) begünstigt wird.

Außerdem wurden bei adipösen Patienten verminderte plasmatische Antithrombin-Spiegel ge- funden (Batist et al. 1983). Antithrombin ist der physiologische Inhibitor aktivierter Gerinnungsfaktoren (v.a. Thrombin und Faktor Xa). Das sehr hohe Thromboembolie-Risiko bei einem angeborenen Antithrombinmangel zeigt die Bedeutung eines reduzierten Antithrombin-Spiegels .

Darüber hinaus zeigen Thrombozyten von Adipösen ein relevant erhöhtes mittleres Volumen (MPV: mean platelet volume). Diese morphologische Auffälligkeit ist mit mehreren funktionellen Veränderungen vergesellschaftet, die in der Summe zu einer gesteigerten Thrombozytenaktivität führen (Anfossi et al. 2010). Alle drei an der primären Hämostase beteiligten Prozesse (Adhäsion, Aktivierung, Aggregation von Thrombozyten) sind bei Adipositas verändert. So ist bei einer vaskulären Verletzung die Adhäsion der Thrombozyten am subendothelialen Kollagen durch eine vermehrte Expression von Adhäsionsmolekülen gesteigert. Die erhöhte Aktivierung von Thrombozyten resultiert unter anderem aus einer gesteigerten enzymatischen Aktivität von Thromboxan $\mathrm{A}_{2}$, einem wichtigen Thrombozytenaktivator (Anfossi et al. 2010). Das aus Fettzellen freigesetzte Adipokin Leptin führt über die Bindung an einen spezifischen thrombozytären Oberflächenrezeptor zur Aktivierung von Thrombozyten. Auch hieraus resultiert eine gesteigerte Synthese von Thromboxan $\mathrm{A}_{2}$, wodurch es zu einer weiteren Zunahme der Thrombozytenaktivierung kommt. Das Resultat ist eine ver- 
mehrte Expression von GP IIb-IIIa-Rezeptoren auf der Oberfläche der Thrombozyten. Über diese Rezeptoren wird die Aggregation der Thrombozyten vermittelt, wobei hauptsächlich Fibrinogen, unter bestimmten Umständen auch der von-Willebrand-Faktor, als Ligand (Bindungsmolekül) fungiert. Leptin wird wegen dieser Eigenschaft auch als proaggregatives Hormon bezeichnet (Anfossi et al. 2010).

Eine weitere Ursache für eine gesteigerte Thrombozytenaktivität bei Adipositas ist das verminderte Ansprechen von Thrombozyten auf Mediatoren, die physiologischerweise zu deren Funktionshemmung beitragen (Anfossi et al. 2010). So reagieren Thrombozyten von Adipösen deutlich schwächer auf Prostacyclin ( $\left.\mathrm{PGI}_{2}\right)$ und NO (Stickstoffmonoxid).

Adipozyten können durch Einfluss auf Thrombozyten die Entstehung von Gerinnungsthromben begünstigen und durch Hemmung der Fibrinolyse im Thrombus den physiologischen Abbau von überschüssigem Fibrin verhindern. Dies führt insgesamt zu einem gesteigerten Thromboserisiko.

Hieraus leitet sich die Frage ab, ob bei Adipositas Thrombozytenaggregationshemmer und Antikoagulanzien zur Thromboseprophylaxe oder Thrombosetherapie in erhöhter Dosierung appliziert werden sollten. Viele Medikamente werden körpergewichtsbezogen dosiert. Dies hängt mit dem veränderten Verteilungsvolumen eines Pharmakons bei unterschiedlichem Körpergewicht zusammen. Hierbei spielt die Pharmakokinetik des Medikaments eine wichtige Rolle. So gibt es lipophile Pharmaka, wie z.B. Diazepam, deren initiale Dosis bei Adipositas erhöht werden muss. Herzglykoside jedoch reichern sich aufgrund ihrer geringen lipophilen Eigenschaft nicht im Fettgewebe sondern hauptsächlich in der Muskulatur an und werden deshalb bei Adipositas nicht höher dosiert. Grundsätzlich kann festgehalten werden, dass vor allem lipophile Pharmaka bei Patienten mit Adipositas dosisangepasst werden müssen.

Im folgenden Abschnitt werden die Dosierungen der einzelnen Antikoagulanzien und Thrombozytenaggregationshemmer hinsichtlich der gewichtsbezogenen Dosierung beschrieben. Es wurden hierzu die aktuellen Fachinformationen der Hersteller und die internationale Literatur herangezogen.

\subsection{Antikoagulation bei Adipositas}

Bei der Beschreibung der verschiedenen Antikoagulanzien und Thrombozytenaggregationshemmer werden vor allem deren Wirkung, Indikation und die unterschiedlichen Dosierungen abhängig vom Kör- pergewicht hervorgehoben. Kontraindikationen und Wechselwirkungen sowie weitere spezifische Informationen werden nicht erwähnt. Hierzu sollten die aktuellen Fachinformationen der Hersteller eingesehen werden.

\subsubsection{Unfraktioniertes Heparin (UFH)}

Heparin ist ein indirekter Thrombin-Inhibitor, der seine antikoagulatorische Wirkung über Antithrombin entfaltet. Antithrombin hemmt aktivierte Gerinnungsfaktoren (v.a. Thrombin und Faktor Xa), wodurch prokoagulatorische Prozesse auf den Ort der vaskulären Verletzung begrenzt werden. UFH haben eine höhere Affinität zu Thrombin als niedermolekulare Heparine.

\section{Standard-Dosierung (Fachinformation)}

- Thromboseprophylaxe: 2-3 x täglich 5.00o I.E. oder $2 \mathrm{x}$ täglich 7.500 I.E. subkutan.

- Therapie der Thrombose und Lungenembolie: Nach einem intravenös verabreichten Bolus von 5.000 I.E. kontinuierliche Zufuhr von ca. 1.0oo I.E. pro Stunde über Perfusor (Ziel: 1,5-2,5-fache Verlängerung der aPTT).

\section{Dosierung bei Adipositas}

Angaben aus der Fachinformation des Herstellers: Die Fachinformation enthält keinen Hinweis auf eine gewichtsadaptierte Dosisanpassung für unfraktioniertes Heparin zur Thromboseprophylaxe oder Therapie der Thrombose und Lungenembolie

Angaben aus der Literatur: Um das Gewicht zu berechnen, auf das die zu verabreichende Heparin-Dosis bei Adipositas bezogen werden soll (Dosierungsgewicht), können folgende Formeln verwendet werden.

Formel zur Berechnung des Gewichts, auf das die Heparin-Dosis bezogen wird

Dosierungsgewicht $=$ Idealgewicht $[\mathrm{kg}]+0,4 \times$ (aktuelles Gewicht [kg] - Idealgewicht [kg])

Das bedeutet für einen adipösen Patienten mit $200 \mathrm{~kg}$ mit einem Idealgewicht von $80 \mathrm{~kg}$ :

Dosierungsgewicht $=80 \mathrm{~kg}+0,4 \times(200-80)=80 \mathrm{~kg}+$ $48=128 \mathrm{~kg}$ (Myziensky 2010).

- Thromboseprophylaxe bei $200 \mathrm{~kg}$ Körpergewicht: Circa 20.0oo IE Heparin pro Tag iv. oder sc.

- Therapie der Venenthrombose: Circa 25.000 bis 35.0oo IE Heparin pro Tag (Effekt über aPTTKontrolle). 
Berechnung des Idealgewichts (IBW) nach

Lemmens 2005

IBW = $22 \times(\text { Körpergröße in } \mathrm{m})^{2}$

\subsubsection{Niedermolekulare Heparine (NMH), z.B. Enoxaparin, Nadroparin, Dalteparin}

Niedermolekulare Heparine sind indirekte Faktor Xaund Thrombin-Inhibitoren, die ihre antikoagulatorische Wirkung über Antithrombin entfalten. Antithrombin hemmt aktivierte Gerinnungsfaktoren, wodurch prokoagulatorische Prozesse auf den Ort einer vaskulären Verletzung begrenzt werden.

Niedermolekulare Heparine werden bei Adipositas gewichtsadaptiertdosiert (Dinwoodey u. Ansell 2006). Eine Erhöhung der Dosierung von NMH für Thromboseprophylaxe sollte ab einem BMI > 40 erwogen werden. Die Dosierung bei therapeutischer Antikoagulation kann auf das aktuelle Gewicht bezogen werden. Ab einem Gewicht von $190 \mathrm{~kg}$ sollte ein Monitoring der Wirkung über die Anti-Xa-Wirkung durchgeführt werden (Nutesco et al. 2009).

\section{Enoxaparin (Clexane ${ }^{\circledR}$ )}

\section{Standard-Dosierung}

(Fachinformation, Stand März 2010)

- Thromboseprophylaxe: Bei hohem perioperativem thromboembolischen Risiko wird die Thromboseprophylaxe mit 1 x täglich 40 mg Enoxaparin subkutan durchgeführt. Die erste Dosis sollte ca. 12 Stunden vor der Operation appliziert werden. Für internistische Patienten mit mittlerem oder hohem Thromboembolierisiko gilt die gleiche Dosierung. Bei Patienten mit stark eingeschränkter Nierenfunktion (Kreatinin-Clearance $<30 \mathrm{ml} / \mathrm{h}$ ) muss die Dosis reduziert werden.

- Therapie der Venenthrombose und Lungenembolie: Hierzu wird Enoxaparin in einer Dosierung von $2 \mathrm{x}$ tgl. $1 \mathrm{mg} / \mathrm{kg}$ Körpergewicht empfohlen.

\section{Dosierung bei Adipositas}

Angaben aus der Fachinformation des Herstellers: Die aktuelle Fachinformation enthält keinen Hinweis auf eine Dosisanpassung zur Thromboseprophylaxe oder Therapie von Thromboembolien bei übergewichtigen und fettleibigen Patienten.

Angaben aus der Literatur: In einer Studie über die Thromboseprophylaxe bei adipösen Patienten mit einem durchschnittlichen Körpergewicht von 135,6 kg bzw. einem BMI von durchschnittlich 48,1 wurde eine Enoxaparin-Dosis von $1 \mathrm{x}$ täglich 0,5 mg/kg Körpergewicht subkutan als effektiv und sicher bewertet (Rondina et al. 2010).

In einer anderen wissenschaftlicher Arbeit über die Anwendung von Enoxaparin bei akutem Koronarsyndrom bzw. Nicht-ST-Hebungsinfarkten wurde eine inverse und stufenweise Dosisanpassung abhängig vom Körpergewicht untersucht (Spinler et al. 2009). In Abhängigkeit vom Körpergewicht wurden folgende Dosierungen von Enoxaparin verabreicht.

\section{Dosis-Anpassung für Enoxaparin an das aktuelle Körpergewicht (Spinler 2009)}

- Patienten mit einem Körpergewicht bis $100 \mathrm{~kg}$ : $1,0 \mathrm{mg} / \mathrm{kg}(2 \times \mathrm{tgl}$.)

- Patienten mit einem Körpergewicht zwischen 101 und $120 \mathrm{~kg}: 0,96 \mathrm{mg} / \mathrm{kg}(2 \times \mathrm{tgl}$.)

- Patienten mit einem Körpergewicht zwischen 121 und $150 \mathrm{~kg}: 0,92 \mathrm{mg} / \mathrm{kg}$ (2 $\mathrm{xtgl}$.)

- Patienten mit einem Körpergewicht über $150 \mathrm{~kg}$ : $0,65 \mathrm{mg} / \mathrm{kg}(2 \times \mathrm{tgl}$.)

Die Studie zeigte bei den Patienten mit einem Körpergewicht über $150 \mathrm{~kg}$ eine erhöhte Blutungsneigung, wenn die vom Hersteller empfohlene Dosierung von $1 \mathrm{mg} / \mathrm{kg}$ Körpergewicht $(2 \mathrm{x}$ tgl.) angewandt wurde, verglichen mit der in der Studie verwendeten Dosis von o, $65 \mathrm{mg} / \mathrm{kg} \mathrm{KG} \mathrm{(2} \mathrm{x} \mathrm{tgl.).}$

Dosierung von niedermolekularem Heparin (hier Enoxaparin ${ }^{\circledR}$ ) angelehnt an das zitierte Stufenschema mit Überprüfung des Effekts auf die Hämostase durch Messung der Anti-Xa-Aktivität.

\section{Nadroparin (Fraxiparin ${ }^{\circledR}$ )}

\section{Standard-Dosierung}

(Fachinformation, Stand März 2010)

- Thromboseprophylaxe: 1 x täglich 2.850 Anti-Xa-Einheiten $(\mathrm{o}, 3 \mathrm{ml})$ subkutan.

- Therapie der Venenthrombose und Lungenembolie: Abhängig vom Körpergewicht $2 \times$ täglich 3.800 $(\mathrm{o}, 4 \mathrm{ml})$ bis 8.550 Anti-Xa-Einheiten $(\mathrm{o}, 9 \mathrm{ml})$ subkutan.

\section{Dosierung bei Adipositas}

- Angaben aus der Fachinformation des Herstellers zur Thromboseprophylaxe:

Bei einem Körpergewicht über $70 \mathrm{~kg}$ soll die Dosis auf $1 \times$ täglich 3.800 Anti-Xa-Einheiten $(0,4 \mathrm{ml})$ erhöht werden. 
- Angaben aus der Fachinformation des Herstellers zur Therapie der Venenthrombose:

Die Therapie erfolgt gewichtsabhängig mit einer Dosierung von jeweils $2 \mathrm{x}$ täglich 7.60o Anti-XaEinheiten (o, $8 \mathrm{ml}$ ) bei einem Körpergewicht zwischen 80 und $89 \mathrm{~kg}$ und $2 \mathrm{x}$ täglich 8.550 Anti-XaEinheiten bei einem Körpergewicht ab go kg.

Angaben aus der Literatur: Die Literaturrecherche in Pubmed zu Nadroparin und Adipositas ergab keine relevanten Informationen, insbesondere keine zu einer Dosisanpassung bei Adipositas.

Dosierung wie vom Hersteller für Patienten mit einem Körpergewicht über $70 \mathrm{~kg}$ (Thromboseprophylaxe) bzw. einem Körpergewicht ab $90 \mathrm{~kg}$ (Therapie der Venenthrombose) empfohlen (s.o.).

\section{Dalteparin (Fragmin ${ }^{\circledR}$ )}

\section{Standard-Dosierung}

(Fachinformation, Stand September 2010)

- Thromboseprophylaxe: In Abhängigkeit vom Thromboserisiko 1 x täglich 2.50o bis 5.00o I.E. subkutan.

- Therapie der Thrombose und Lungenembolie: Die Therapie erfolgt gewichtsadaptiert mit

1 $1 \times$ täglich 200 I.E. pro kg Körpergewicht subkutan oder:

v 1 x täglich 12.500 I.E. subkutan bei $57-68 \mathrm{~kg}$ Körpergewicht

v 1 x täglich 15.000 I.E: subkutan bei $69-82 \mathrm{~kg}$ Körpergewicht

\section{Dosierung bei Adipositas}

- Angaben aus der Fachinformation des Herstellers zur Thromboseprophylaxe:

Die aktuelle Fachinformation enthält keine Angaben über die Dosierung von Dalteparin zur Thromboseprophylaxe bei übergewichtigen und fettleibigen Patienten.

- Angaben aus der Fachinformation des Herstellers zur Therapie der Venenthrombose:

1. $1 \times$ tgl 15.00o I.E. subkutan bei 69-82 kg Körpergewicht

- $1 \mathrm{x}$ tgl. 18.00o I.E: subkutan bei über 83 kg Körpergewicht.

Angaben aus der Literatur: Dalteparin sollte gewichtsbezogen verabreicht werden. Ab einem Körpergewicht über $190 \mathrm{~kg}$ sollte ein Monitoring über die Anti-Xa-Aktivität erfolgen (Nutesco et al. 2009).
Dosierung wie vom Hersteller für Patienten mit einem Körpergewicht über $83 \mathrm{~kg}$ empfohlen, d.h. maximal 1 x tgl. 18.000 I.E. subkutan.

\subsubsection{Danaparoid (Orgaran ${ }^{\circledR}$ )}

Danaparoid ist ein Heparinoid (Gemisch aus Glykosaminoglykane) mit den Hauptinhaltsstoffen Heparansulfat und Dermatansulfat. Danaparoid wird vor allem zur Prophylaxe und Therapie der Heparin-induzierten Thrombozytopenie Typ II (HIT-II) eingesetzt.

\section{Standard-Dosierung (Fachinformation, Stand April 2008)}

- Thromboseprophylaxe: 2 x täglich 750 Anti-Xa-Einheiten subkutan.

- Thromboseprophylaxe bei HIT-II mit geringem Thromboembolierisiko: 2 x täglich 750 Anti-Xa-Einheiten subkutan.

- Thromboseprophylaxe bei HIT-II mit akuter HIT-II: 3 x täglich 750-1.250 Anti-Xa-Einheiten subkutan.

- Therapie der Heparin-induzierten Thrombozytopenie:

- Initial Verabreichung eines intravenösen Bolus von 2.250 Anti-Xa-Einheiten.

- Nach dem Bolus kontinuierliche Verabreichung von Orgaran ${ }^{\circledR}$ :

400 Anti-Xa-Einheiten pro Stunde über 4 Stunden, dann 300 Anti-Xa-Einheiten pro Stunde über 4 Stunden, anschließend 150-200 AntiXa-Einheiten pro Stunde für 5-7 Tage.

\section{Dosierung bei Adipositas}

- Angaben aus der Fachinformation des Herstellers:

- Patienten mit einem Gewicht über 9o kg erhalten zur Therapie einer HIT-II einen intravenösen Bolus von 3.750 Anti-Xa-Einheiten Danaparoid.

- Anschließend wird das gleiche Dosisschema wie bei Normgewichtigen angewandt. Nach dem Bolus kontinuierliche Verabreichung von Danaparoid:

400 Anti-Xa-Einheiten pro Stunde über 4 Stunden, dann 300 Anti-Xa-Einheiten pro Stunde über 4 Stunden, anschließend 150-200 AntiXa-Einheiten pro Stunde für 5-7 Tage.

Die aktuelle Fachinformation enthält keine Angaben über die Dosierung von Danaparoid zur Thromboseprophylaxe bei übergewichtigen und fettleibigen Patienten. 
Angaben aus der Literatur: Die Literaturrecherche in Pubmed zu Danaparoid und Adipositas ergab keine relevanten Informationen, insbesondere keine zu einer Dosisanpassung bei Adipositas.

Dosierung wie vom Hersteller für Patienten mit einem Körpergewicht über $90 \mathrm{~kg}$ empfohlen (s.o.).

\subsubsection{Lepirudin (Refludan ${ }^{\circledR}$ )}

Lepirudin ist ein direkter Thrombin-Inhibitor und wird vor allem zur Prophylaxe und Therapie der Heparin-induzierten Thrombozytopenie Typ II (HIT II) eingesetzt. Aufgrund seines Wirkungsmechanismus ist Lepirudin vom Antithrombin-Spiegel unabhängig. Besonders zu beachten ist, dass Lepirudin größtenteils renal eliminiert wird und deshalb eine deutliche Dosisreduktion bei eingeschränkter Nierenfunktion vorzunehmen ist. Die Wirkung von Lepirudin auf die Hämostase kann mittels der aPTT überprüft und gesteuert werden.

\section{Standard-Dosierung}

(Fachinformation, Stand November 2008)

Bei normaler Nierenfunktion wird eine Anfangsdosis von o, $15 \mathrm{mg} / \mathrm{kg}$ Körpergewicht pro Stunde empfohlen. Vorab kann je nach Schweregrad des Krankheitsbildes ein Bolus von o,4 mg/kg Körpergewicht verabreicht werden. Bis zu einem Körpergewicht von $110 \mathrm{~kg}$ richtet sich die Dosierung nach dem aktuellen Körpergewicht.

\section{Dosierung bei Adipositas}

Angaben aus der Fachinformation des Herstellers: Auch bei einem Gewicht $>110 \mathrm{~kg}$ sollte die für $110 \mathrm{~kg}$ errechnete Dosis nicht überschritten werden. Die Wirkung von Refludan kann über die Kontrolle der aPTT überprüft werden.

Angaben aus der Literatur: In einem publizierten Fallbericht wurde Refludan zur Behandlung einer Lungenembolie bei Heparin-Resistenz bei einem Patienten mit einem Körpergewicht von $145 \mathrm{~kg}$ eingesetzt (Imman u. Gerlach 2009). Initial wurde eine Dosierung von $2 x$ $50 \mathrm{mg}$ Lepirudin subkutan verabreicht. Die Dosis wurde nach wenigen Tagen, basierend auf der gemessen aPTT auf $2 \times 25 \mathrm{mg}$ subkutan und schließlich auf $1 \times 25 \mathrm{mg}$ subkutan reduziert. Ein Grund für die Dosisreduktion war auch eine Verschlechterung der Nierenfunktion mit einem Anstieg des Serumkreatinins von initial 1,3 auf $1,6 \mathrm{mg} / \mathrm{dl}$. Der Patient konnte ohne Auftreten einer tie- fen Venenthrombose (Duplexsonografie-Nachweis) nach Hause entlassen werden.

Anwendung der Standard-Dosierung unter Berücksichtigung der Maximaldosierung, errechnet für $110 \mathrm{~kg}$ Körpergewicht.

\subsubsection{Desirudin $\left(\operatorname{Revasc}^{\circledR}\right.$ )}

Desirudin ist ein direkter Thrombin-Inhibitor und wird vor allem zur Sekundär-Prophylaxe nach abgelaufener Heparin-induzierter Thrombozytopenie Typ II eingesetzt. Das Präparat ist derzeit in Deutschland nicht verfügbar.

\subsubsection{Argatroban (Argatra $\left.{ }^{\circledR}\right)$}

Argatroban ist ein direkter Thrombin-Inhibitor, der vor allem zur Prophylaxe und Therapie der Heparininduzierten Thrombozytopenie Typ II (HIT II) eingesetzt wird.

\section{Standard-Dosierung \\ (Fachinformation, Stand Dezember 2009)}

Die Dosierung von Argatroban erfolgt gewichtsbezogen: Nach Beginn mit der vom Hersteller empfohlenen Anfangsdosierung von 1-2 $\mu \mathrm{g} / \mathrm{kg} / \mathrm{min}$ und Kontrolle des Effekts über die aPTT ( 2 Stunden nach Therapie-Beginn) kann die Argatroban-Dosis je nach Ziel-aPTT gesteigert werden, maximal auf $10 \mu \mathrm{g} / \mathrm{kg} /$ min. Die maximale empfohlene Behandlungsdauer beträgt 14 Tage. Bei kritisch Kranken, Patienten mit Lebererkrankungen und nach Herzoperationen wird eine reduzierte Anfangsdosis von o, $5 \mu \mathrm{g} / \mathrm{kg} / \mathrm{min}$ verabreicht.

\section{Dosierung bei Adipositas}

Angaben aus der Fachinformation des Herstellers: Die aktuelle Fachinformation enthält keine spezifischen Angaben zur Dosierung von Argatroban bei übergewichtigen und fettleibigen Patienten.

Angaben aus der Literatur: Die Literaturrecherche in Pubmed zu Argatroban und Adipositas ergab keine relevanten Informationen, insbesondere keine zu einer Dosisanpassung bei Adipositas.

Anwendung der Standard-Dosierung mit Überprüfung des Effekts auf die Hämostase durch Messung der aPTT. 


\subsubsection{Dabigatran (Pradaxa ${ }^{\circledR}$ )}

Dabigatran ist ein direkter Thrombin-Inhibitor und wird derzeit zur Thromboseprophylaxe nach Hüftund Kniegelenkersatz eingesetzt.

\section{Standard-Dosierung}

(Fachinformation, Stand Januar 2011)

$1 \mathrm{x}$ täglich $220 \mathrm{mg}$ (die erste postoperative Dosis beträgt no mg und sollte 1-4 Stunden nach Operationsende verabreicht werden).

\section{Dabigatran-Dosierung bei Adipositas}

Der Hersteller hält eine Dosisanpassung für Patienten mit einem Körpergewicht über $110 \mathrm{~kg}$ für nicht erforderlich, weist jedoch auf die begrenzte Erfahrung bzgl. der Dosierung für diese Patienten hin.

Angaben aus der Literatur: Bei Adipositas wird eine Erhöhung der Dosis nicht für notwendig erachtet (Stangier 2009).

\section{Anwendung der Standard-Dosierung.}

\subsubsection{Fondaparinux (Arixtra ${ }^{\circledR}$ )}

Fondaparinux ist ein indirekter Faktor Xa-Inhibitor, der seine antikoagulatorische Wirkung über Antithrombin entfaltet. Fondaparinux wird derzeit vor allem zur Thromboseprophylaxe nach Hüft- und Kniegelenkersatz eingesetzt.

\section{Standard-Dosierung}

(Fachinformation, Stand August 2010)

- Thromboseprophylaxe: $1 \times$ tgl. 2,5 mg subkutan(1. Injektion 6 Stunden postoperativ).

- Für internistische Patienten mit einem erhöhten Thromboembolierisiko wird auch eine Dosis von $1 \mathrm{x}$ täglich 2,5 mg subkutan empfohlen.

\section{Dosierung bei Adipositas}

Die aktuelle Fachinformation enthält keine Angaben über die Dosierung von Fondaparinux zur Thromboseprophylaxe bei übergewichtigen und fettleibigen Patienten.

Auswahl eines Alternativpräparates zur Thromboseprophylaxe (beispielsweise ein niedermolekulares Heparin).

\subsubsection{Rivaroxaban (Xarelto ${ }^{\circledR}$ )}

Rivaroxaban ist ein direkter Faktor Xa-Inhibitor und wird derzeit zur Thromboseprophylaxe nach Hüftund Kniegelenkersatz eingesetzt.

Standard-Dosierung (Fachinformation Stand Januar 2011)

- $1 \mathrm{x}$ tgl. $10 \mathrm{mg}$, wobei die erste Dosis 6-1o Stunden nach Operationsende verabreicht werden soll.

\section{Rivaroxaban-Dosierung bei Adipositas}

Angaben aus der Fachinformation des Herstellers: Die aktuelle Fachinformation weist darauf hin, dass für Rivaroxaban keine Dosisanpassung bzgl. des Körpergewichts erforderlich ist.

Angaben aus der Literatur: Die Literaturrecherche in Pubmed zu Rivaroxaban und Adipositas ergab keine relevanten Informationen, insbesondere keine zu einer Dosisanpassung bei Adipositas.

\section{Anwendung der Standard-Dosierung.}

\subsubsection{Phenprocoumon (Marcumar ${ }^{\circledR}$, Falithrom ${ }^{\circledR}$ )}

Phenprocoumon ist ein Vitamin K-Antagonist mit den Hauptindikationsgebieten Vorhofflimmern und Herzklappenersatz sowie Sekundärprophylaxe nach Thrombose und Lungenembolie.

\section{Standard-Dosierung (Fachinformation Stand April 2010)}

Die Dosierung erfolgt individuell, angepasst an die vom behandelnden Arzt festgelegte Ziel-INR bzw. den gewünschten Quick-Wert.

\section{Dosierung bei Adipositas}

Die aktuelle Fachinformation enthält keine spezifischen Angaben zur Dosierung von Phenprocoumon bei übergewichtigen und fettleibigen Patienten.

Angaben aus der Literatur: Die Wirkung von Phenprocoumon wird stark vom Körpergewicht beeinflusst, sodass bei adipösen Patienten höhere Dosierungen appliziert bzw. kürzere Applikationsintervalle eingehalten werden müssen, um die Ziel-INR zu erreichen (Meyer zu Schwabedissen et al. 2006).

Dosierung wie üblich an Ziel-INR bzw. Ziel-Quick-Wert anpassen, wobei zu erwarten ist, dass kürzere Applikations-Intervalle bzw. höhere Einzeldosen notwendig werden. 


\subsubsection{Acenocoumarol (Sintrom ${ }^{\circledR}$ )}

Acenocoumarol ist ein Vitamin K-Antagonist mit den Hauptindikationsgebieten Vorhofflimmern und Herzklappenersatz sowie Sekundärpropylaxe nach Thrombose und Lungenembolie. Acenocoumarol unterscheidet sich von Phenprocoumon vor allem durch seine kürzere Wirkungsdauer.

\section{Standard-Dosierung (Fachinformation Stand April 2008)}

Die Dosierung erfolgt individuell, angepasst an die vom behandelnden Arzt festgelegte Ziel-INR bzw. den gewünschten Quick-Wert.

\section{Dosierung bei Adipositas}

Die aktuelle Fachinformation enthält keine spezifischen Angaben zur Dosierung von Acenocoumarol bei übergewichtigen und fettleibigen Patienten.

Angaben aus der Literatur: Die Literaturrecherche in Pubmed zu Acenocoumarol und Adipositas ergab keine relevanten Informationen, insbesondere keine zu einer Dosisanpassung bei Adipositas.

Dosierung wie üblich an Ziel-INR bzw. Ziel-Quick-Wert anpassen, wobei zu erwarten ist, dass kürzere Applikations-Intervalle bzw. höhere Einzeldosen notwendig werden.

\subsubsection{Warfarin (Coumadin ${ }^{\circledR}$ )}

Warfarin ist ein Vitamin K-Antagonist mit den Hauptindikationsgebieten Vorhofflimmern und Herzklappenersatz sowie Sekundärpropylaxe nach Thrombose und Lungenembolie. Warfarin unterscheidet sich von Phenprocoumon vor allem durch seine kürzere Wirkungsdauer.

\section{Standard-Dosierung}

(Fachinformation Stand August 2008)

Die Dosierung erfolgt individuell, angepasst an die vom behandelnden Arzt festgelegte Ziel-INR bzw. den gewünschten Quick-Wert.

\section{Dosierung bei Adipositas}

Die aktuelle Fachinformation enthält keine spezifischen Angaben zur Dosierung von Warfarin bei übergewichtigen und fettleibigen Patienten.

Angaben aus der Literatur: Die Literaturrecherche in Pubmed zu Warfarin und Adipositas ergab keine relevanten
Informationen, insbesondere keine zu einer Dosisanpassung bei Adipositas.

Dosierung wie üblich an Ziel-INR bzw. Ziel-Quick-Wert anpassen, wobei zu erwarten ist, dass kürzere Applikations-Intervalle bzw. höhere Einzeldosen notwendig werden.

\section{Azetylsalizylsäure/ASS (ASS 100 ${ }^{\circledR}$ )}

Azetylsalizylsäure (ASS) gehört zur Cruppe der Thrombozytenfunktionshemmer. ASS hemmt irreversibel das Enzym Zyklooxygenase, wodurch in Thrombozyten die Synthese des Thrombozyten-Aktivators Thromboxan $\mathrm{A}_{2}$ gehemmt wird.

\section{Standard-Dosierung (Fachinformation Stand Juli 2009)} $1 \mathrm{x}$ täglich $100 \mathrm{mg}$ ASS

\section{Dosierung bei Adipositas}

Die aktuelle Fachinformation enthält keine spezifischen Angaben zur Dosierung von ASS bei übergewichtigen und fettleibigen Patienten.

Angaben aus der Literatur: Für Thrombozyten von Adipösen konnte eine höhere Basisaktivität festgestellt werden. Nach Verabreichung der Standarddosis (hier $81 \mathrm{mg}$ ASS täglich) wurde die Aktivität der Thrombozyten von Adipösen deutlich geringer gehemmt als die von Normalgewichtigen (Bordeaux 2010).

In den aktuell gültigen, internationalen Empfehlungen (Patrono 2008) wird nicht speziell auf den Aspekt des Körpergewichts eingegangen. Es wird jedoch betont, dass ASS in einer Dosierung zwischen 50 und $100 \mathrm{mg}$ effizient ist und dass der Effekt, z.B. beim Einsatz zur Sekundärprophylaxe nach TIA (transitorisch ischämische Attacke) durch eine Dosissteigerung nicht erhöht wird. Es wird deshalb in diesen Leitlinien eine Dosis von täglich 50-100 mg empfohlen.

Für (schwer) Adipöse gilt zur optimalen Dosis von ASS: Durchführung einer Laboruntersuchung zur Überprüfung der Wirksamkeit: Multiplate (R) oder PFA-100 (R).

\subsubsection{Clopidogrel (Plavix ${ }^{\circledR}$, Iscover ${ }^{\circledR}$ )}

Clopidogrel ist ein Thrombozytenfunktionshemmer, der seinen Effekt vor allem durch Blockade des thrombozytären Oberflächenrezeptors für den Thrombozyten-Aktivator ADP (Adenosin-Diphosphat) entfaltet. 


\section{Standard-Dosierung}

(Fachinformation Stand Januar 2011)

- 1 x täglich $75 \mathrm{mg}$.

- Bei den meisten Indikationen (akuter Myokardinfarkt, Stent-Implantation) wird zuvor eine Aufsättigungsdosis von $300 \mathrm{mg}$ verabreicht.

\section{Dosierung bei Adipositas}

Angaben aus der Fachinformation des Herstellers: Die aktuelle Fachinformation enthält keine spezifischen Angaben zur Dosierung von Clopidogrel bei übergewichtigen und fettleibigen Patienten.

Angaben aus der Literatur: In einer im Jahr 2008 publizierten Studie wurde festgestellt, dass bei einem Körpergewicht über $83 \mathrm{~kg}$ mehr Patienten als sogenannte „low responder" identifiziert werden konnten. Der hemmende Effekt von Clopidogrel auf Thrombozyten konnte durch Verdopplung der täglichen Dosis auf $150 \mathrm{mg}$ bei einer deutlich höheren Patientenzahl erzielt werden (Aleil 2008). Unter einer Dosis von täglich 75 mg Clopidogrel war die thromboembolische Komplikationsrate bei Patienten mit Adipositas (BMI > 30) innerhalb des ersten Jahres nach Insertion eines Drug Eluting Stent nahezu doppelt so hoch wie bei normalgewichtigen Patienten. Es wurde hieraus abgeleitet, eine gewichtsabhängige Dosierung von Clopidogrel zu erwägen (Sarno 2010).

Der hemmende Effekt von Clopidogrel auf Thrombozyten kann über die Messung der Thrombozytenfunktion, beispielsweise mit dem Multiplate ${ }^{\circledR}$-Gerät, objektiviert werden. So kann individuell die Dosierung festgelegt werden. Steht eine solche Messmethode nicht zur Verfügung und liegen keine blutungsfördernden Faktoren vor, ist bei (schwer) Adipösen eine Erhöhung der Clopidogrel-Dosis zu erwägen.

\subsubsection{Prasugrel (Efient ${ }^{\circledR}$ )}

Prasugrel ist ein Thrombozytenfunktionshemmer, der seinen Effekt vor allem durch Blockade des thrombozytären Oberflächenrezeptors für den Thrombozyten-Aktivator ADP (Adenosin-Diphosphat) entfaltet.

\section{Standard-Dosierung}

(Fachinformation Stand September 2009)

- Aufsättigungsdosis 1 x 60 mg,

- anschließend Erhaltungsdosis 1 x 10 mg täglich.

\section{Dosierung bei Adipositas}

Die aktuelle Fachinformation enthält keine spezifischen Angaben zur Dosierung von Prasugrel bei übergewichtigen und fettleibigen Patienten.

Angaben aus der Literatur: Die Literaturrecherche in Pubmed zu Prasugrel und Adipositas ergab keine relevanten Informationen, insbesondere keine zu einer Dosisanpassung bei Adipositas.

\section{Anwendung der Standard-Dosierung!}

\subsubsection{Alprostadil (Prostavasin ${ }^{\circledR} 20 \mu \mathrm{g}$ )}

Alprostadil ist ein Prostaglandin, das neben einer profunden vasodilatatorischen Wirkung auf das Kapillargebiet auch einen hemmenden Effekt auf die Thrombozytenfunktion hat. Es wird zur Verbesserung der Durchblutung bei peripherer arterieller Verschlusskrankheit Stadium III und IV eingesetzt.

\section{Standard-Dosierung (Fachinformation Stand Februar 2011)}

Angaben aus der Fachinformation des Herstellers:

n Intraarterielle Kurzinfusion: 1/2 Ampulle Prostavasin ${ }^{\circledR} 20 \mu \mathrm{g}$ (10 $\mu \mathrm{g}$ Alprostadil) wird über 6o120 Minuten mittels Infusionspumpe i.a. infundiert ( $1 \times$ tgl.).

- Intraarterielle Dauerinfusion: o,1-o,6 ng/kg KG/ min über 12 Stunden mittels Infusionspumpe (entsprechend $1 / 4-1 \frac{1}{2}$ Ampullen Prostavasin ${ }^{\circledR} 20 \mu \mathrm{g}$ ).

\section{Dosierung bei Adipositas}

Die aktuelle Fachinformation enthält keine spezifischen Angaben zur Dosierung von Alprostadil bei übergewichtigen und fettleibigen Patienten.

Angaben aus der Literatur: Die Literaturrecherche in Pubmed zu Alprostadil und Adipositas ergab keine relevanten Informationen, insbesondere keine zu einer Dosisanpassung bei Adipositas.

\section{Anwendung der Standard-Dosierung.}

\subsubsection{Alteplase/rt-PA (Actilyse ${ }^{\circledR}$ )}

Alteplase (rt-PA) ist ein Fibrinolytikum und wird zur Lyse venöser und arterieller Thromboembolien eingesetzt. 


\section{Standard-Dosierung}

(Fachinformation Stand Februar 2007)

- Bei akutem Myokardinfarkt werden insgesamt $100 \mathrm{mg}$ Alteplase verabreicht: Nach einem $15 \mathrm{mg}$-Bolus werden $50 \mathrm{mg}$ über 30 Minuten und anschließend $35 \mathrm{mg}$ über 6o Minuten appliziert.

- Bei akuter Lungenembolie werden insgesamt $100 \mathrm{mg}$ Alteplase verabreicht: Nach einem $10 \mathrm{mg}$-Bolus werden go mg Alteplase über 2 Stunden intravenös appliziert.

\section{Dosierung bei Adipositas}

Angaben aus der Fachinformation des Herstellers: Die aktuelle Fachinformation enthält keine spezifischen Angaben zur Dosierung von Alteplase bei übergewichtigen und fettleibigen Patienten.

Angaben aus der Literatur: Die Literaturrecherche in Pubmed zu Alteplase und Adipositas ergab keine relevanten Informationen, insbesondere keine zu einer Dosisanpassung bei Adipositas.

\section{Anwendung der Standard-Dosierung.}

\subsubsection{Antithrombin (z.B. Kybernin ${ }^{\circledR}$ )}

Antithrombin ist physiologischer Inhibitor aktivierter Gerinnungsfaktoren wie Thrombin und Faktor Xa. Alle Heparine sowie Fondaparinux und Danaparoid entfalten ihren antikoagulatorischen Effekt über Antithrombin. Der hereditäre AntithrombinMangel gehört zu den Thrombophilie-Formen mit dem höchsten thromboembolischen Risiko. Antithrombin kann bei Mangel-Zuständen als Konzentrat zugeführt werden. Bei adipösen Patienten wurden verminderte plasmatische Antithrombin-Spiegel gefunden (Batist et al. 1983).

\section{Standard-Dosierung}

(Fachinformation Stand Januar 2009)

- Erforderliche Einheiten = Körpergewicht $[\mathrm{kg}] \times$ (100 - aktuelle AT III-Aktivität [\%]) x 2/3

- 1I.E. Antithrombin $/ \mathrm{kg}$ Körpergewicht erhöht die Antithrombin III-Aktivität um ca. 1,5\%

\section{Dosierung bei Adipositas}

Die aktuelle Fachinformation enthält keine spezifischen Angaben zur Dosierung von Antithrombin bei übergewichtigen und fettleibigen Patienten.
Angaben aus der Literatur: Die Literaturrecherche in Pubmed zu Antithrombin und Adipositas ergab keine relevanten Informationen, insbesondere keine zu einer Dosisanpassung bei Adipositas.

\section{Anwendung der Standard-Dosierung.}

\section{Fazit}

Durch die stetige Zunahme der Anzahl übergewichtiger Patienten im Klinikalltag gewinnt das Thema Adipositas eine immer größere Bedeutung. Bei der Durchsicht der Literatur zu diesem Buchkapitel fiel auf, dass gerade im Bereich der Dosierung von Medikamenten für dieses Patientenkollektiv präzise Angaben, auch in den Fachinformationen der Hersteller, eher selten sind.

Künftige wissenschaftliche Untersuchungen, insbesondere Dosisfindungsstudien, sollten daher auch dieses wachsende Patientenkollektiv stärker berücksichtigen. Es wäre wünschenswert, wenn hierbei einheitliche, international gültige Definitionen für Übergewicht, Fettleibigkeit und extreme Fettleibigkeit angewendet würden. So könnten diesen Informationen auch in die Leitlinien der Fachgesellschaften einfließen.

\section{Literatur}

Aleil B, Jacquemin L, De Poli F, Zaehringer M, Collet IP, Montalescot G, Cazenave IP, Dickele MC, Monassier IP, Gachet C (2008) Clopidogrel $150 \mathrm{mg} /$ day to overcome low responsiveness in patients undergoing elective percutaneous coronary intervention: results from the VASP-02 (Vasodilator-Stimulated Phosphoprotein-02) randomized study. JACC Cardiovasc Interv 6, 631-638

Anfossi G, Russo I, Doronzo G, Pomero A, Trovati M (2010) Adipocytokines in atherothrombosis: focus on platelets and vascular smooth muscle cells. Mediators Inflamm 174341

Batist G, Bothe A Jr, Bern M, Bistrian BR, Blackburn GL (1983) Low antithrombin III in morbid obesity: return to normal with weight reduction. J Parenter Enteral Nutr 7, 447-449

Binder BR, Christ G, Gruber F, Grubic N, Hufnagl P, Krebs M, Mihaly I, Prager GW. (2002) Plasminogen activator inhibitor 1: physiological and pathophysiological roles. News Physiol Sci 17, 56-61

Bordeaux BC, Qayyum R, Yanek LR, Vaidya D, Becker LC, Faraday N, Becker DM (2010) Effect of obesity on platelet reactivity and response to low-dose aspirin. Prev Cardiol 13, 56-62

Dinwoodey DL, Ansell JE (2006) Heparins, low-molecular-weight heparins, and pentasaccharides. Clin Geriatr Med 22, 1-15

Inman KR, Gerlach AT (2009) Use of subcutaneous lepirudin in an obese surgical intensive care unit patient with heparin resistance. Ann Pharmacother 43, 1714-1718

Lemmens HJ, Brodsky JB, Bernstein DP (2005) Estimating ideal body weight--a new formula. Obes Surg 15, 1082-1083 
Loskutoff D), Samad F (1998) The adipocyte and hemostatic balance in obesity: studies of PAl-1. Arterioscler Thromb Vasc Biol 18, 1-6 Meyer zu Schwabedissen C, Mevissen V, Schmitz F, Woodruff S, Langebartels G, Rau T, Zerres K, Hoffmann R, Ortlepp IR (2006) Obesity is associated with a slower response to initial phenprocoumon therapy whereas $\mathrm{CYP}_{2} \mathrm{C}_{9}$ genotypes are not. Eur I Clin Pharmacol 62, 713-720

Myzienski AE, Lutz MF, Smythe MA (2010) Unfractionated heparin dosing for venous thromboembolism in morbidly obese patients: case report and review of the literature. Pharmacotherapy 30,324

Nafiu 00, Shanks AM, Hayanga A), Tremper KK, Campbell DA Jr (2011) The impact of high body mass index on postoperative complications and resource utilization in minority patients. I Natl Med Assoc 103, 9-15

Nutescu EA, Spinler SA, Wittkowsky A, Dager WE (2009) Low-molecular-weight heparins in renal impairment and obesity: available evidence and clinical practice recommendations across medical and surgical settings. Ann Pharmacother 43, 1064-1083

Patrono C, Baigent C, Hirsh J, Roth G; American College of Chest Physicians (2008) Antiplatelet drugs: American College of Chest Physicians Evidence-Based Clinical Practice Guidelines (8th Edition). Chest 133, 199S-233S
Rondina MT, Wheeler M, Rodgers GM, Draper L, Pendleton RC (2010) Weight-based dosing of enoxaparin for VTE prophylaxis in morbidly obese, medically-Ill patients. Thromb Res 125, 220-223

Sarno G, Garg S, Onuma Y, Buszman P, Linke A, Ischinger T, Klauss V, Eberli F, Corti R, Wijns W, Morice MC, di Mario C, van Geuns RI, Eerdmans P, Garcia-Garcia HM, van Es GA, Goedhart D, de Vries T, Jüni P, Meier B, Windecker S, Serruys P (2010) The impact of body mass index on the one year outcomes of patients treated by percutaneous coronary intervention with Biolimus- and Sirolimus-eluting stents (from the LEADERS Trial). Am I Cardiol 105, 475-479

Spinler SA, Ou FS, Roe MT, Gibler WB, Ohman EM, Pollack CV, Alexander KP, Peterson ED (2009) Weight-based dosing of enoxaparin in obese patients with non-ST-segment elevation acute coronary syndromes: results from the CRUSADE initiative. Pharmacotherapy 29, 631-638

Stangier I, Clemens A (2009) Pharmacology, pharmacokinetics, and pharmacodynamics of dabigatran etexilate, an oral direct thrombin inhibitor. Clin Appl Thromb Hemost 15 Suppl 1, 9S-16S

The Acute Respiratory Distress Syndrome Network (2000) Ventilation with lower tidal volumes as compared with traditional tidal volumes for acute lung injury and the acute respiratory distress syndrome. N Engl | Med 342, 1301-1308

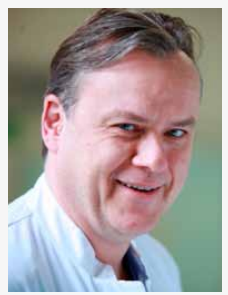

\section{Dr. med. Claus Steuernagel}

Nach dem Medizinstudium in Mainz (Approbation 1986, Promotion 1987) Weiterbildung zum Facharzt für Anästhesie am Bundeswehrzentralkrankenhaus in Koblenz und an den Horst-Schmidt-Kliniken Wiesbaden. Seit Oktober 1998 Oberarzt am Elisabeth-Krankenhaus Essen in der Klinik für Anästhesie, Intensivmedizin und Schmerztherapie mit dem Zuständigkeitsbereich operative Intensivmedizin. Im Jahr 2007 Erwerb der Zusatzweiterbildung Hämostaseologie, seit 2010 Vertreter des Klinikdirektors Prof. Dr. med. Klaus Lewandowski. 